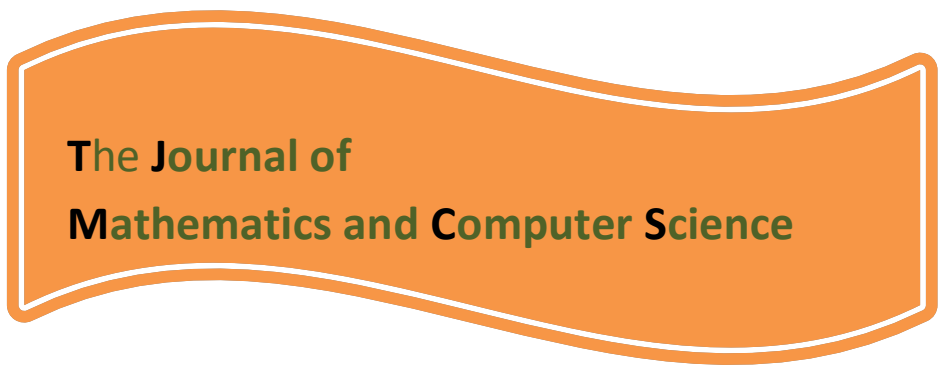

Available online at

http://www.TJMCS.com

The Journal of Mathematics and Computer Science Vol. 4 No.1 (2012) 60 - 70

\title{
Multiple solution to (p,q)-Laplacian systems with concave nonlinearities
}

\author{
G.A. Afrouzi and M. Bai \\ Department of mathematics, Faculty of Mathematical Sciences, \\ University of Mazandaran, Babolsar, Iran \\ E-mail: afrouzi@umz.ac.ir \\ bai_marjan@yahoo.com
}

Received: November 2011, Revised: February 2012

Online Publication: May 2012

\section{Abstract}

In this paper we study the (p,q)-Laplacian systems with concave nonlinearities. Using some asymptotic behavior $f$ at zero and infinity, three nontrivial solutions are established.

Keywords: Nonlinear boundary value problem, Concave nonlinearity, (p,q)-Laplacian systems, Variational method, Multiple solutions.

\section{AMS Subject classification: $35 j 65$}

\section{Introduction}

In this paper, we consider problems

$$
\left\{\begin{array}{lr}
-\Delta_{p} u=\lambda|u|^{p-2} u+f_{u}(x, u, v) & \text { in } \Omega \\
-\Delta_{q} v=\lambda|v|^{q-2} v+f_{v}(x, u, v) & \text { in } \Omega \\
u=v=0 & \text { in } \partial \Omega
\end{array}\right.
$$

Where $\Omega \subset R^{N},(N \geq 1)$ is a bounded with smooth domain and $F \in C^{1}\left(\bar{\Omega} \times R^{2}, R\right)$. The functional corresponding to problems (1.1) is

$J_{\lambda}(u, v)=\frac{1}{p} \int_{\Omega}|\nabla u|^{p} d x+\frac{1}{q} \int_{\Omega}|\nabla \mathrm{v}|^{\mathrm{q}} \mathrm{dx}-\frac{\lambda}{\mathrm{p}} \int_{\Omega}|\mathrm{u}|^{\mathrm{p}} \mathrm{dx}-\frac{\lambda}{\mathrm{q}} \int_{\Omega}|\mathrm{v}|^{\mathrm{q}} \mathrm{dx}-\int_{\Omega} \mathrm{F}(\mathrm{x}, \mathrm{u}, \mathrm{v}) \mathrm{dx}$ Let $W=W_{0}^{1, P}(\Omega) \times W_{0}^{1, q}(\Omega)$ with the norm 


$$
\|(u, v)\|=\|\nabla u\|_{p}+\|\nabla v\|_{q}=\left(\int_{\Omega}|\nabla u|^{p} d x\right)^{\frac{1}{p}}+\left(\int_{\Omega}|\nabla \mathrm{v}|^{q} d x\right)^{\frac{1}{q}} .
$$

It is well known operator $-\Delta$ has a sequence of eigenvalues $\left\{\lambda_{k}\right\}$ satisfying $0<\lambda_{1}<\lambda_{2}<\cdots<\lambda_{k} \rightarrow+\infty$. For general $(p, q) \in(1,+\infty),\left(-\Delta_{p},-\Delta_{q}\right)$ has a smallest eigenvalue, i.e., the principle value, $\lambda_{1}$, which is positive, isolated, simple (see[2]) and admit the following variational characterization

$$
\lambda_{1}=\inf _{0 \neq u, v \in W_{0}^{1, p}(\Omega) \times W_{0}^{1, q}(\Omega)} \frac{\int_{\Omega}|\nabla u|^{p} d x+\int_{\Omega}|\nabla v|^{q} d x}{\int_{\Omega}|u|^{p} d x+\int_{\Omega}|v|^{q} d x}
$$

Furthermore, the $\lambda_{1}$ - eigenfunctions do not change in $\Omega$, and by the maximum principle we may suppose that $\phi_{1}>0$ is a $\lambda_{1}$ - eigenfunction. There are many paper concerned with the resonance problem. In [7] L. Shi proved that there exists $\lambda^{*}>0$ such that pLaplacian multiple solutions for a class of (p,q)-Laplacian systems (1.1). Consider the following conditions hold:

(i) $\quad f(0,0)=0$.

(ii) $f \in C^{1}\left(\Omega \times R^{2}, R\right)$ and $f^{\prime}(0,0)>\lambda_{1}$.

(iii) For some positive integer $k \geq 1$,

$$
\lim _{|(s, t)| \rightarrow-\infty} \frac{f(x, s, t)}{|s|^{p}+|t|^{q}}<\lambda_{1} \leq \lambda_{k}<\lim _{|(s, t)| \rightarrow+\infty} \frac{f(x, s, t)}{|s|^{p}+|t|^{q}}<\lambda .
$$

In this paper we extend this result to the case $1<p, q<+\infty$; Furtheremore, here the $\lim _{|(s, t)| \rightarrow+\infty} \frac{f(x, s, t)}{|s|^{p}+|t|^{q}} \in\left(\lambda_{k}, \lambda_{k+1}\right)$ relaxed to

$$
\mu_{2} \leq \liminf _{|(s, t)| \rightarrow \infty} \frac{f(x, s, t)}{|s|^{p-2} s+|t|^{q-2} t} \leq \limsup _{|(s, t)| \rightarrow \infty} \frac{f(x, s, t)}{|s|^{p-2} s+|t|^{q-2} t} \leq \mu_{3}
$$

Where $\mu_{2}, \mu_{3} \in\left(\lambda_{1},+\infty\right)$.

Our main result is as follows.

Theorem 1.1. Assume that $f \in\left(\bar{\Omega} \times R^{2}, R\right)$ and $f(x, 0,0)=0$ a.e. If the following conditions hold

(i) There exists constant $\mu_{0}>\lambda_{1}$ such that

$$
\lim _{|(s, t)| \rightarrow \infty} \frac{f(x, s, t)}{|s|^{p-2} s+|t|^{q-2} t} \geq \mu_{0} \quad \text { Uniformly for a.e. } x \in \Omega \text {; }
$$

(ii) There exist constants $\mu_{1}, \mu_{2}, \mu_{3}>0$ with $\mu_{1}<\lambda_{1}<\mu_{2}$ such that

$$
\lim _{|(s, t)| \rightarrow \infty} \frac{f(x, s, t)}{|s|^{p-2} s+|t|^{q-2} t} \leq \mu_{1}
$$




$$
\mu_{2} \leq \liminf _{|(s, t)| \rightarrow+\infty} \frac{f(x, s, t)}{|s|^{p-2} s+|t|^{q-2} t} \leq \underset{|(s, t)| \rightarrow+\infty}{\lim u p} \frac{f(x, s, t)}{|s|^{p-2} s+|t|^{q-2} t} \leq \mu_{3}
$$

Hold uniformly for a.e. $x \in \Omega$, then there exist such that problem (1.1) admits at least three nontrivial solutions for $\lambda \in\left(0, \lambda^{*}\right)$.

\section{2 proof of the main result}

Define the functional $J_{\lambda}: W_{0}^{1, p}(\Omega) \times W_{0}^{1, q}(\Omega) \rightarrow \mathrm{R}$ by

$$
J_{\lambda}(u, v)=\frac{1}{p} \int_{\Omega}|\nabla u|^{p} d x+\frac{1}{q} \int_{\Omega}|\nabla v|^{q} d x-\frac{\lambda}{p} \int_{\Omega}|\mathbf{u}|^{p} d x-\frac{\lambda}{q} \int_{\Omega}|v|^{q} d x-\int_{\Omega} F(x, u, v) d x
$$

Clearly, $J_{\lambda} \in C^{1}\left(W_{0}^{1, p}(\Omega) \times W_{0}^{1, q}(\Omega), \mathrm{R}\right)$. It is obviouse that the critical points of correspond to the weak solutions of problem (1.1).

Lemma 2.1. Assume that the assumptions of theorem 1.1 hold. Then the functional $J_{\lambda}(u, v)$ satisfies the (PS) condition.

Proof: Assume that $\left\{\left(u_{n}, v_{n}\right)=z_{n}\right\}_{n \in N} \subset W_{0}^{1, p}(\Omega) \times W_{0}^{1, q}(\Omega)$ is a (PS) sequence, i.e., for some $M>0$,

$$
\left|J_{\lambda}\left(u_{n}, v_{n}\right)\right| \leq M, \quad \nabla J_{\lambda}\left(u_{n}, v_{n}\right) \rightarrow 0 \text { as } n \rightarrow \infty .
$$

It suffices to prove that $\left\{\left(u_{n}, v_{n}\right)=z_{n}\right\}$ is bounded in $W_{0}^{1, p}(\Omega) \times W_{0}^{1, q}(\Omega)$. In fact, if not, we may assume by contradiction that there exist a sequence of $\left\{\left(u_{n}, v_{n}\right)=z_{n}\right\}$ with $\left\|\left(u_{n}, v_{n}\right)\right\| \rightarrow+\infty$ and $\{\varepsilon\}$ with $\varepsilon_{n} \rightarrow 0$ in

$W_{0}^{-1, p^{\prime}}(\Omega) \times W_{0}^{-1, q^{\prime}}(\Omega)$ such that

$$
-\Delta_{p} u_{n}=-\lambda\left|u_{n}\right|^{p-2} u+f_{u}\left(x, u_{n}, v_{n}\right) \quad \text { in } \quad W_{0}^{-1, p^{\prime}}(\Omega)
$$

Taking $-u_{n}^{-}$as test function in (2.2), we obtain

$$
\left\|\nabla u_{n}^{-}\right\|_{p}^{p}=\int_{\Omega} \lambda\left|u_{n}^{-}\right|^{p} d x-\int_{\Omega} f_{u}\left(x, u_{n}, v_{n}\right) u_{n}^{-} d x-\int_{\Omega} \varepsilon_{n} u_{n}^{-} d x .
$$

Similarly,

$$
\left\|\nabla v_{n}^{-}\right\|_{q}^{q}=\int_{\Omega} \lambda\left|v_{n}^{-}\right|^{q} d x-\int_{\Omega} f_{v}\left(x, u_{n}, v_{n}\right) v_{n}^{-} d x-\int_{\Omega} \varepsilon_{n} v_{n}^{-} d x
$$


In view of (4.1), for any $\varepsilon \in\left(0, \lambda_{1}-\mu_{1}\right)$, there exists $C=C(\varepsilon)>0$ such that

$$
f(x, s, t) \geq\left(\mu_{1}+\varepsilon\right)\left(|s|^{p-2} s+|t|^{q-2} t\right)-C \quad \forall s, t<0 \quad \text { a.e } e, x \in \Omega \quad .
$$

Then by the sobolev embedding and Poincare inequality there exist $C_{1}, C_{2}>0$

Such that

$$
\begin{gathered}
\left\|\nabla u_{n}^{-}\right\|_{p}^{p}+\left\|\nabla v_{n}^{-}\right\|_{q}^{q} \leq \\
\int_{\Omega} \lambda\left(\left|u_{n}^{-}\right|^{p}+\left|v_{n}^{-}\right|^{q}\right) d x+\int_{\Omega}\left(\mu_{1}+\varepsilon\right)\left(\left|u_{n}^{-}\right|^{p}+\left|v_{n}^{-}\right|^{q}\right) d x-\int_{\Omega}\left(C-\varepsilon_{n}\right)\left(u_{n}^{-}+v_{n}^{-}\right) d x \\
\leq C_{1} \int_{\Omega} \lambda\left(\left\|u_{n}^{-}\right\|^{p}+\left\|v_{n}^{-}\right\|^{q}\right) d x+\int_{\Omega} \frac{\mu_{1+\varepsilon}}{\lambda_{1}}\left(\left\|u_{n}^{-}\right\|^{p}+\left\|v_{n}^{-}\right\|^{q}\right) d x \\
+C_{2} \int_{\Omega}\left(\left\|u_{n}^{-}\right\|+\left\|v_{n}^{-}\right\|\right) d x .
\end{gathered}
$$

Hence by $\mu_{1}+\varepsilon<\lambda_{1}$, it follows that $\left\{\left(u_{n}^{-}, v_{n}^{-}\right)=z_{n}^{-}\right\} \subset W_{0}^{1, p}(\Omega) \times W_{0}^{1, q}(\Omega)$ is bounded. For any $n$, we take $\psi_{n, k}=-\left(\left(u_{n}+v_{n}\right) k\right)^{-}$with $k>0$ as test function in (2.2), using again (2.3), we get

$$
\begin{aligned}
& \int_{\Omega}\left|\nabla \psi_{n, k}\right|^{p} d x \leq \\
& -\int_{\Omega} \lambda\left(\left|u_{n}^{-}\right|^{p-2}+\left|v_{n}^{-}\right|^{q-2}\right) \psi_{n, k} d x+\int_{\Omega}\left(\mu_{1}+\varepsilon\right)\left(\left|u_{n}^{-}\right|^{p-2}+\left|v_{n}^{-}\right|^{q-2}\right) \psi_{n, k} d x \\
& -\int_{\Omega}\left(C-\varepsilon_{n}\right) \psi_{n, k} d x .
\end{aligned}
$$

We can obtain that $\left\{\left\|\left(u_{n}^{-}, v_{n}^{-}\right)\right\|_{\infty}\right\}$ is bounded. By the standard regularity theory (see [4]), it follow that there exists $C_{3}>0$ such that, for every $n, u_{n} \in C^{1, \sigma}(\bar{\Omega})$ for some $\sigma>0$ and

$$
\left\|\left(\nabla u_{n+} \nabla v_{n}\right)\right\|_{\infty} \leq C_{3}\left(1+\left\|\left(u_{n}+v_{n}\right)\right\|_{\infty}\right) .
$$

Thus by $\left\|\left(u_{n}, v_{n}\right)\right\|_{\infty} \rightarrow+\infty$ it follows that $\left\|\left(u_{n}^{+}, v_{n}^{+}\right)\right\|_{\infty} \rightarrow+\infty$.

We may assume that $z_{n}=\left\|\left(u_{n}, v_{n}\right)\right\| \rightarrow \infty$ as $n \rightarrow \infty$. Define $\hat{u}_{n}=\frac{u_{n}}{z_{n}}, \hat{v}_{n}=\frac{v_{n}}{z_{n}}$. 
Denote $g(x, s, t)=-\lambda\left(|s|^{p-2} s+|t|^{q-2} t\right)+f(x, s, t)$. In view of $(2.1)$, for all $\phi \in W_{0}^{1, p}(\Omega) \times$ $W_{0}^{1, q}(\Omega)$, we have

$$
\int_{\Omega}\left[\left|\nabla \hat{u}_{n}\right|^{p-2} \nabla \hat{u}_{n} \nabla \phi-\frac{g_{u\left(x, u_{n}, v_{n}\right)}}{z_{n}^{p-1}} d t\right] \rightarrow 0
$$

Since $g$ is countinous and $\left\|\left(u_{n}^{-}, v_{n}^{-}\right)\right\|_{\infty}$ is uniformly bounded, using (1.3)-(1.5) and (2.5), there exist constant $C_{4}, C_{5}>0$ and $\varepsilon \in\left(0, \mu_{2}-\lambda_{1}\right)$ such that

$$
\left(\mu_{2}-\varepsilon\right)\left|\hat{u}_{n}(x)\right|^{p-1}-\frac{C_{4}}{\left\|z_{n}\right\|_{\infty}^{p-1}} \leq \frac{g\left(x, u_{n}, v_{n}\right)}{\left\|z_{n}\right\|_{\infty}^{p-1}} \leq\left(\mu_{3}+\varepsilon\right)\left|\hat{u}_{n}\right|^{p-1}+\frac{C_{5}}{\left\|z_{n}\right\|_{\infty}^{p-1}}
$$

Hold uniformly for a.e. $x \in \Omega$. In a similarly way, we get $\hat{u}_{n} \rightarrow \hat{v}_{0}$. By the regularity theory (see [4]), there exists a constant $M_{2}>0$ such that, for every $n$, $\left\|\left(\hat{u}_{n}, \hat{v}_{n}\right)\right\|_{C^{1, \sigma}} \leq M_{2}$, set $w_{n}=\frac{z_{n}}{\left\|z_{n}\right\|_{\infty}}$. Then by the compact imbedding of $C^{1, \sigma}(\bar{\Omega})$

into $C^{1}(\bar{\Omega})$, passing to a subsequence if possible, we have

$$
w_{n} \rightarrow w_{0} \quad \text { in } \quad C^{1}(\bar{\Omega})
$$

With $\left\|\hat{u}_{0}\right\|_{\infty}=1$, then $\left(\hat{u}_{n}, \hat{v}_{n}\right)$ is bounded Which $\left\|\hat{u}_{n}\right\|_{1, p}+\left\|\hat{v}_{n}\right\|_{1, q}=1$.

Using again that $\left\|\left(\hat{u}_{n}, \hat{v}_{n}\right)\right\|$ is bounded and $\hat{u}_{n}=\frac{u_{n}^{+}-u_{n}^{-}}{\left\|z_{n}\right\|_{\infty}}$, we can see that $\hat{u}_{0} \geq 0$ and $\hat{u}_{0} \not \equiv 0$, similarly for $\hat{v}_{n}=\frac{v_{n}^{+}-v_{n}^{-}}{\left\|z_{n}\right\|_{\infty}}$ and we can see that $\hat{u}_{0} \geq 0$ and $\hat{u}_{0} \not \equiv 0$.

Denote $\alpha_{n}(x)=\frac{g\left(x, u_{n}, v_{n}\right)}{\left\|z_{n}\right\|_{\infty}^{p-1}}$. By (2.7) and (2.8) if follows that there exists $\alpha \in L^{\infty}(\Omega)$ satisfying

$$
\mu_{2}-\varepsilon \leq \alpha(x) \leq \mu_{3}+\varepsilon
$$

Such that

$$
\alpha_{n} \rightarrow \alpha\left(\left|\hat{u}_{0}\right|^{p-2} u_{0}+\left|\hat{v}_{0}\right|^{p-2} v_{0}\right) \quad \text { weakly in } L^{\infty}(\Omega)
$$

By (2.6), (2.8), (2.10) we obtain

$$
\int_{\Omega}\left|\nabla \hat{u}_{0}\right|^{p-2} \nabla u_{0} \nabla \phi d x=\int_{\Omega}\left[\alpha(x)\left|\hat{u}_{0}\right|^{q-2} u_{0}\right] \phi d x
$$

For every $\phi \in W_{0}^{1, p}(\Omega) \times W_{0}^{1, q}(\Omega)$. Consequently, similarly

$$
\int_{\Omega}\left|\nabla \hat{v}_{0}\right|^{p-2} \nabla v_{0} \nabla \phi d x=\int_{\Omega}\left[\alpha(x)\left|\hat{v}_{0}\right|^{q-2} v_{0}\right] \phi d x
$$


$\left(\hat{u}_{0}, \hat{v}_{0}\right)$ is a nontrivial solution of

$$
\left\{\begin{aligned}
-\Delta_{p} w_{0} & =\alpha(x) w_{0}^{p-1} & & \text { in } \Omega \\
-\Delta_{q} w_{0} & =\alpha(x) w_{0}^{q-1} & & \text { in } \Omega \\
w_{0} & =0 & & \text { on } \partial \Omega
\end{aligned}\right.
$$

By the maximum principle of vazquez's [9], it follows that $w_{0}(x)>0$ for $x \in \Omega$.

Furthermore, there is a positive constant $\delta>0$ and $\varphi=\left(\varphi_{1}, \varphi_{2}\right)$ such that

$$
\delta \varphi \leq w_{0} \quad \text { on } \partial \Omega
$$

By (2.9),(2.11) and $\mu_{2}>\lambda_{1}$, for any $\varepsilon \in\left(0, \frac{\mu_{2}-\lambda_{1}}{2}\right)$, we get

$$
-\Delta_{p} w_{0}>\left(\lambda_{1}, \lambda_{1}+\varepsilon\right) w_{0}^{p-1}
$$

And

$$
-\Delta_{q} w_{0}>\left(\lambda_{1}, \lambda_{1}+\varepsilon\right) w_{0}^{q-1} .
$$

Take $\psi=\left(\psi_{1}, \psi_{2}\right)$ and $\psi=\delta \varphi$ and $\mu \in\left(\lambda_{1}, \lambda_{1}+\varepsilon\right)$. Then we have

$$
\begin{gathered}
-\Delta_{p} \psi=\lambda_{1} \psi^{p-1} \leq \mu \psi^{p-1} \\
\text { and } \\
-\Delta_{q} \psi=\lambda_{1} \psi^{q-1} \leq \mu \psi^{q-1} .
\end{gathered}
$$

By (2.12) and (2.13), by the method of sub and supersolution, for any $\varepsilon>0$ small enough, we can obtain a solution $(\bar{u}, \bar{v}) \in\left[\psi, w_{0}\right]$ of the following problems

$$
\left\{\begin{array}{lr}
-\Delta_{p} u=\mu u^{p-1} & \text { in } \Omega \\
-\Delta_{q} v=\mu v^{q-1} & \text { in } \Omega \\
u=v=0 & \text { on } \partial \Omega
\end{array}\right.
$$

However, this is contrary to this fact that $\lambda_{1}$ is isolated. Hence $\left\{\left\|\left(u_{n}^{+}, v_{n}^{+}\right)\right\|\right\}$is also uniformly bounded. Thus by (2.4) we can see that the sequence $\left\{\|\left(u_{n}, v_{n} \|\right\}\right.$ is uniformly bounded. Then using standard arguments we can see that $J_{\lambda}$ satisfies the (PS) condition. This completes the proof.

Define 


$$
f_{+}(x, s, t)=\left\{\begin{array}{rr}
f(x, s, t) & t, s \geq 0 \\
0 & t, s \leq 0
\end{array}\right.
$$

Define the corresponding functional $J_{\lambda(u, v)}^{+}: W_{0}^{1, p}(\Omega) \times W_{0}^{1, q}(\Omega) \rightarrow R$ as follows.

$$
J_{\lambda}^{+}(u, v)=\frac{1}{p} \int_{\Omega}|\nabla u|^{p} d x+\frac{1}{q} \int_{\Omega}|\nabla v|^{q} d x-\frac{\lambda}{p} \int_{\Omega}\left|u^{+}\right|^{p} d x-\frac{\lambda}{q} \int_{\Omega}\left|v^{+}\right|^{q} d x-\int_{\Omega} F_{+}(x, u, v) d x,
$$

Where $\nabla F=\left(f_{u}, f_{v}\right)$. Obviously, $J_{\lambda}^{+} \in C^{1}\left(W_{0}^{1, p}(\Omega) \times W_{0}^{1, q}(\Omega), R\right)$. Similarly, define

$$
f_{-}(x, s, t)=\left\{\begin{array}{cc}
f(x, s, t) & t, s \leq 0 \\
0 & t, s \geq 0
\end{array}\right.
$$

Define the corresponding functional $J_{\lambda(u, v)}^{-}: W_{0}^{1, p}(\Omega) \times W_{0}^{1, q}(\Omega) \rightarrow R$ as follows.

$$
J_{\lambda}^{-}(u, v)=\frac{1}{p} \int_{\Omega}|\nabla u|^{p} d x+\frac{1}{q} \int_{\Omega}|\nabla v|^{q} d x-\frac{\lambda}{p} \int_{\Omega}\left|u^{-}\right|^{p} d x-\frac{\lambda}{q} \int_{\Omega}\left|v^{-}\right|^{q} d x-\int_{\Omega} F_{-}(x, u, v) d x
$$

Where $\nabla F=\left(f_{u}, f_{v}\right)$. It is easily seen that $J_{\lambda}^{-} \in C^{1}\left(W_{0}^{1, p}(\Omega) \times W_{0}^{1, q}(\Omega), R\right)$.

Using similar arguments as in the proof of lemma 2.1, we obtain the following result.

Lemma 2.2. The functional $\mathrm{J}_{\lambda}^{ \pm}$satisfies the (PS) condition.

To prove Theorem 1.1, we prove some preliminary results as follows.

Lemma 2.3. If $\left(u^{ \pm}, v^{ \pm}\right)$is a local minimizer of $\mathrm{J}_{\lambda}^{ \pm}$, then it is also a local minimizer of $\mathrm{J}_{\lambda}$.

Proof: By Theorem 1.1 of Garcia Azorero, Peral Alonso and Manfredi[5], we just need to show that $\left(u^{ \pm}, v^{ \pm}\right)$is a local minimazer of $J_{\lambda}$ in the $C^{1}$ topology. By the assumption it follow that $\left(u^{ \pm}, v^{ \pm}\right)$is a $C_{0}^{1}(\bar{\Omega})$-local minimize of $\mathrm{J}_{\lambda}^{ \pm}$i.e., there exists $\rho_{1}>0$ such that

$$
\mathrm{J}_{\lambda}^{ \pm}\left(u^{ \pm}, v^{ \pm}\right) \leq \mathrm{J}_{\lambda}^{ \pm}(\mathrm{u}, \mathrm{v}), \quad \forall u \in B_{\rho_{1}}\left(u^{ \pm}, v^{ \pm}\right)
$$

Where $B_{\rho_{1}}\left(u^{ \pm}, v^{ \pm}\right)=\left\{(u, v) \in C_{0}^{1}(\bar{\Omega}):\left\|(u, v)-\left(u^{ \pm}, v^{ \pm}\right)\right\|_{C^{1}}<\rho_{1}\right\}$. By (1.4), (1.5), we can see that $f$ is of p-linear growth [5]. Then, for $(u, v) \in B_{\rho_{1}}\left(u^{ \pm}, v^{ \pm}\right)$, we obtain 


$$
\begin{aligned}
J_{\lambda}(u, v)-J_{\lambda}\left(u^{ \pm}, v^{ \pm}\right)=J_{\lambda}(u, v)-\mathrm{J}_{\lambda}^{ \pm}\left(u^{ \pm}, v^{ \pm}\right) \\
\quad \geq \frac{\lambda}{p} \int_{\Omega}\left[|(u, v)|^{p}-\left|\left(u^{ \pm}, v^{ \pm}\right)\right|^{p}\right] d x \\
+\frac{\lambda}{q} \int_{\Omega}\left[|(u, v)|^{q}-\left|\left(u^{ \pm}, v^{ \pm}\right)\right|^{q}\right] d x-\int_{\Omega}\left[F(x, u, v)-F_{ \pm}(x, u, v)\right] d x \\
=\frac{\lambda}{p} \int_{\Omega}\left|\left(u^{\mp}, v^{\mp}\right)\right|^{p} d x+\frac{\lambda}{q} \int_{\Omega}\left|\left(u^{\mp}, v^{\mp}\right)\right|^{q} d x-\int_{\Omega} F_{\mp}(x, u, v) d x \\
\geq \frac{\lambda}{p} \int_{\Omega}\left|\left(u^{\mp}, v^{\mp}\right)\right|^{p} d x+\frac{\lambda}{q} \int_{\Omega}\left|\left(u^{\mp}, v^{\mp}\right)\right|^{q} d x \\
-C\left(\int_{\Omega}\left|\left(u^{\mp}, v^{\mp}\right)\right|^{P} d x+\int_{\Omega}\left|\left(u^{\mp}, v^{\mp}\right)\right|^{q} d x\right) \\
\geq\left[\frac{\lambda}{q}-C\left\|\left(u^{\mp}, v^{\mp}\right)\right\|_{\infty}^{p-q}\right] \int_{\Omega}\left|\left(u^{\mp}, v^{\mp}\right)\right|^{q} d x \\
+\left[\frac{\lambda}{p}-C\left\|\left(u^{\mp}, v^{\mp}\right)\right\|_{\infty}^{q-p}\right] \int_{\Omega}\left|\left(u^{\mp}, v^{\mp}\right)\right|^{p} d x
\end{aligned}
$$

Note $\rho_{1} \rightarrow 0$ implies $\left\|\left(u^{-}, v^{-}\right)\right\|_{\infty} \rightarrow 0$, together with $1<p, q<+\infty$, we can see that there exists $\rho_{2}>0$ small enough such that

$J_{\lambda}\left(u^{ \pm}, v^{ \pm}\right) \leq J_{\lambda}(u, v), \quad \forall(u, v) \in B_{\rho_{2}}\left(u^{ \pm}, v^{ \pm}\right)$,

Where $B_{\rho_{2}}\left(u^{ \pm}, v^{ \pm}\right)=\left\{(u, v) \in C_{0}^{1}(\bar{\Omega}):\left\|(u, v)-\left(u^{ \pm}, v^{ \pm}\right)\right\|_{C^{1}}<\rho_{2}\right\}$. This completes the proof.

Lemma 2.4. 0 is a local minimize of $J_{\lambda}^{ \pm}$and $J_{\lambda}$ for $\lambda>0$.

Proof: we just consider the case of $J_{\lambda}$. The other cases can be treated similarly. As shown in the proof of lemma 2.3, it suffices to prove that 0 is a local minimizer of $J_{\lambda}$ in the $C^{1}$ topology. In fact, for $(u, v) \in C_{0}^{1}(\bar{\Omega})$, we have 


$$
\begin{aligned}
\mathrm{J}_{\lambda}^{ \pm}\left(u^{ \pm}, v^{ \pm}\right) \geq & \frac{\lambda}{p} \int_{\Omega}|(u, v)|^{p} d x+\frac{\lambda}{q} \int_{\Omega}|(u, v)|^{q} d x-\int_{\Omega} F(x, u, v) d x \\
& \geq \frac{\lambda}{p} \int_{\Omega}|(u, v)|^{p} d x+\frac{\lambda}{q} \int_{\Omega}|(u, v)|^{q} d x-C\left(\int_{\Omega}|u|^{p} d x+\int_{\Omega}|v|^{q} d x\right) \\
& \geq\left[\frac{\lambda}{p}-C\|u\|_{\infty}^{q-p}\right] \int_{\Omega}|u|^{p} d x+\left[\frac{\lambda}{q}-C\|u\|_{\infty}^{p-q}\right] \int_{\Omega}|v|^{q} d x
\end{aligned}
$$

If we define $B_{\rho_{3}}(0,0)=\left\{(u, v) \in C_{0}^{1}(\bar{\Omega}):\|(u, v)\|_{C^{1}}<\rho_{3}\right\}$, where $\rho_{3} \in\left(0,\left(\frac{\lambda}{c_{q}}\right)^{\frac{1}{p-q}},\left(\frac{\lambda}{c_{p}}\right)^{\frac{1}{q-p}}\right)$, then it follows that

$$
J_{\lambda}(u, v) \geq 0, \quad \forall(u, v) \in B_{\rho_{3}}(0,0) .
$$

The proof is complete.

Lemma 2.5. There exist $\lambda^{*}, t_{1}, t_{2}>0$ such that, for $\lambda \in\left(0, \lambda^{*}\right)$

$$
J_{\lambda}\left( \pm t_{1} \phi_{1}, \pm t_{2} \phi_{2}\right)<0
$$

Proof: By (1.3)-(1.5), for any given $\varepsilon>0$ and $r \in\left(p, \frac{p n}{n-p}\right)$ if $n>p ; r \in(p,+\infty)$

If $1 \leq n \leq p$, there exist $C>0$ such that

$$
\left.\left.\left|p F(x, z)-\mu_{3}\right| z\right|^{p}|\leq \varepsilon| z\right|^{p}+C|z|^{r} .
$$

Then, taking $\varepsilon<\mu_{3}-\lambda_{1}$, we have $J_{\lambda}\left(t_{1} \phi_{1}, t_{2} \phi_{2}\right)=$

$\frac{\left|t_{1}\right|^{p}}{p}\left\|\phi_{1}\right\|^{p}+\frac{\left|t_{2}\right|^{q}}{q}\left\|\phi_{2}\right\|^{q}+\frac{\left|t_{1}\right|^{p}}{p} \lambda \int_{\Omega} \phi_{1}^{p} d x+\frac{\left|t_{2}\right|^{q}}{q} \lambda \int_{\Omega} \phi_{2}^{q} d x-\int_{\Omega} F\left(t_{1} \phi_{1}, t_{2} \phi_{2}\right) d x \leq$ $\frac{\left|t_{1}\right|^{p}}{p}\left\|\phi_{1}\right\|^{p}+\frac{\left|t_{2}\right|^{q}}{q}\left\|\phi_{2}\right\|^{q}+\frac{\left|t_{2}\right|^{q}}{q} \lambda \int_{\Omega} \phi_{2}^{q} d x-\frac{\left|t_{1}\right|^{p}}{p} \mu_{3} \int_{\Omega} \phi_{1}^{p} d x+\frac{\left|t_{1}\right|^{p}}{p} \varepsilon \int_{\Omega} \phi_{1}^{p} d x+$ $\frac{\left|t_{1}\right|^{r}}{p} C \int_{\Omega} \phi_{1}^{r} d x=\left[\lambda_{1}-\mu_{3}+\varepsilon\right] \frac{\left|t_{1}\right|^{p}}{p} \int_{\Omega} \phi_{1}^{p} d x+\frac{\left|t_{2}\right|^{q}}{q} \int_{\Omega} \phi_{2}^{q} d x+\frac{\left|t_{1}\right|^{r}}{p} C \int_{\Omega} \phi_{1}^{r} d x \leq$ $-\left(\frac{\mu_{3}-\varepsilon}{\lambda_{1}}-1\right) \frac{\left|t_{1}\right|^{p}}{p}\left\|\phi_{1}\right\|^{p}+C\left(\lambda\left|t_{1}\right|^{q-p}+\left|t_{1}\right|^{r-p}\right)\left\|\phi_{1}\right\|^{p}$

Define $\varphi(z)=\lambda z^{q-p}+z^{r-p} \quad$ for $\quad z \geq 0$, where $\delta \equiv \frac{1}{p}\left(\frac{\mu_{3}-\varepsilon}{\lambda_{1}}-1\right)>0$.

Then $\varphi^{\prime}(z)=\lambda(q-p) z^{q-p-1}+(r-p-1) z^{r-p-1}$. 
It is easily seen that $\varphi^{\prime}\left(z_{0}\right)=0$ if $\mathrm{z}_{0}=\left(\frac{\lambda(\mathrm{p}-\mathrm{q})}{\mathrm{r}-\mathrm{p}-1}\right)^{\frac{1}{\mathrm{r}-q}}$. denote $\delta_{0}=\frac{p-q}{r-p-1}$. Then we have $\varphi\left(z_{0}\right)=\left[\delta_{0}^{\frac{q-p}{r-q}}+\delta_{0}^{\frac{r-p}{r-q}}\right] \lambda^{\frac{r-p}{r-q}}$.

Hence if taking $|t|=\mathrm{z}_{0}$, there exists $\lambda^{*}>0$ such that if $\lambda<\lambda^{*}$ then

$$
C \varphi\left(z_{0}\right)<\frac{1}{p}\left(\frac{\mu_{3}-\varepsilon}{\lambda_{1}}-1\right) .
$$

Thus we can see that (2.14) hold for $\lambda \in\left(0, \lambda^{*}\right)$ if we take $t_{1}=z_{0}$.

Proof of theorem 1.1. By lemma 2.4, 0 is a local minimizer of $J_{\lambda}^{ \pm}$and $J_{\lambda}$ with $J_{\lambda}^{ \pm}(0,0)=$ $J_{\lambda}(0,0)=0$. In view of lemma 2.5 , there exist $t_{1}, t_{2}, \lambda^{*}>0$ such that, for

$$
\lambda \in\left(0, \lambda^{*}\right){ }_{W_{0}^{1, p}(\Omega) \times W_{0}^{1, q}(\Omega)} J_{\lambda}^{ \pm}(u, v) \leq J_{\lambda}\left( \pm t_{1} \phi_{1}, \pm t_{2} \phi_{2}\right)<0
$$

Then $J_{\lambda}^{ \pm}$has a nontrivial critical point $\left(u^{ \pm}, v^{ \pm}\right)$of mountain pass type with $J_{\lambda}^{ \pm}\left(u^{ \pm}, v^{ \pm}\right)>0$, which implies that $\left(u^{ \pm}, v^{ \pm}\right)$is a weak solution of the following (p,q)-Laplacian

$$
\left\{\begin{array}{lr}
-\Delta_{p} u=\lambda\left|u^{ \pm}\right|^{p-2}+f_{u}^{ \pm}(x, u, v) & \text { in } \Omega \\
-\Delta_{q} v=\lambda\left|v^{ \pm}\right|^{q-2}+f_{v}^{ \pm}(x, u, v) & \text { in } \Omega \\
u=v=0 & \text { on } \partial \Omega
\end{array}\right.
$$

By the weak maximum principle we can see that $\left( \pm u_{1}^{ \pm}, \pm v_{1}^{ \pm}\right) \geq 0$ in $\Omega$, which implies that $\left(u_{1}^{ \pm}, v_{1}^{ \pm}\right)$is also a solution of system (1.1) and

$J_{\lambda}\left(u^{ \pm}, v^{ \pm}\right)=J_{\lambda}^{ \pm}\left(u^{ \pm}, v^{ \pm}\right)$. In addition, by the fatter of (1.4) it follows that the functional $J_{\lambda}^{-}$ is coercive on $W_{0}^{1, p}(\Omega) \times W_{0}^{1, q}(\Omega)$ and hence bounded below. Combing with (2.15) implies that $J_{\lambda}^{-}$has a nontrivial global minimizer

$\left(u_{2}^{-}, v_{2}^{-}\right)$with $J_{\lambda}^{-}\left(u_{2}^{-}, v_{2}^{-}\right)<0$. Then by lemma 2.3 we can see that $\left(u_{2}^{-}, v_{2}^{-}\right)$is a local minimizer $J_{\lambda}$. Thus (1.1) has at least nontrivial solutions $\left(u_{1}^{-}, v_{1}^{-}\right),\left(u_{2}^{-}, v_{2}^{-}\right),\left(u_{1}^{+}, v_{1}^{+}\right)$.

\section{References}

[1] A. Ambrosetti, H. Brezis, C. Cerami, Combined effects of concave and covex nonlinearities in some elliptic problems, J. Funct. Anal. 122 (1994) 519-543. 
[2] G. A. Afrouzi, S. Mahdavi, Z. Naghizadeh, Existence of multiple solutions for a class of (p,q)-Laplacian systems, Nonlinear Anal. 72 (2010) 2243-2250.

[3] G. A. Afrouzi, and M. Bai, Elliptic P-Laplacian equations with indefinite concave nonlinearities near the origin, ATAM 7/1 (2012) 167 51-57.

[4] G. M. Lieberman, Boundary regularity for solutions to semilinear elliptic problems with combined nonlinearities, J. Differential Equations 185 (2002) 200-224.

[5] J.P. Garcia Azorero, I. Peral Alonso, J.J. Manfredi, Sobolev versus Holder local minimizers and global multiplicity for some quasilinear elliptic equations, Commun. Contemp. Math. 2 (2000) 385-404.

[6] J. Vazquez, A strong maximum principle for some quasilinear elliptic equations, A ppl. Math. Optim. 12 (1984) 191-202.

[7] L. Shi. X. Chang, Multiple solutions to p-Laplacian problems with concave nonlinearities, J. Math. Anal. Appl. 363 (2010) 155-160.

[8] T. F. Wu, On semilinear elliptic equations involving concave-convex nonlinearities and sign-changing weight function, J. Math. Anal. Appl. 318 (2006) 263-270.

[9] Xiao-Xiao Zhao, Chun-Lei Tang, Resonance problems for (p,q)-Laplacian systems, Nonlinear Anal. 72 (2010) 1019-1030. 